\title{
YTHDF2 reduction fuels inflammation and vascular abnormalization in hepatocellular carcinoma
}

\author{
Jiajie Hou ${ }^{1,2,3,4^{*}+}$, He Zhang ${ }^{1,5+}$, Jun Liu ${ }^{6 \dagger}$, Zhenjun Zhao ${ }^{1}$, Jianye Wang ${ }^{1}$, Zhike Lu ${ }^{6}$, Bian Hu${ }^{7}$, Jiankui Zhou' \\ Zhicong Zhao', Mingxuan Feng ${ }^{1}$, Haiyan Zhang ${ }^{4,8}$, Bin Shen ${ }^{9}$, Xingxu Huang ${ }^{7}$, Beicheng Sun ${ }^{2}$, Chuan He $\mathrm{H}^{6,10^{*}}$ and \\ Qiang Xia ${ }^{1 *}$
}

\begin{abstract}
Background: Dynamic $N^{6}$-methyladenosine $\left(\mathrm{m}^{6} \mathrm{~A}\right)$ modification was previously identified as a ubiquitous posttranscriptional regulation that affected mRNA homeostasis. However, the $\mathrm{m}^{6} \mathrm{~A}$-related epitranscriptomic alterations and functions remain elusive in human cancer. Here we aim to identify the profile and outcome of $\mathrm{m}^{6} \mathrm{~A}$ methylation in hepatocellular carcinoma (HCC).

Results: Using liquid chromatography-tandem mass spectrometry and $\mathrm{m}^{6} \mathrm{~A}$-immunoprecipitation in combination with high-throughput sequencing, we determined the $m^{6} \mathrm{~A}-\mathrm{mRNA}$ levels in human HCC. Human HCC exhibited a characteristic gain of $\mathrm{m}^{6} \mathrm{~A}$ modification in tandem with an increase of mRNA expression, owing to YTH domain family 2 (YTHDF2) reduction. The latter predicted poor classification and prognosis of HCC patients, and highly correlated with HCC $\mathrm{m}^{6} \mathrm{~A}$ landscape. YTHDF2 silenced in human HCC cells or ablated in mouse hepatocytes provoked inflammation, vascular reconstruction and metastatic progression. Mechanistically, YTHDF2 processed the decay of $\mathrm{m}^{6} \mathrm{~A}$-containing interleukin 11 (IL11) and serpin family E member 2 (SERPINE2) mRNAs, which were responsible for the inflammation-mediated malignancy and disruption of vascular normalization. Reciprocally, YTHDF2 transcription succumbed to hypoxia-inducible factor-2a (HIF-2a). Administration of a HIF-2a antagonist (PT2385) restored YTHDF2-programed epigenetic machinery and repressed liver cancer.
\end{abstract}

Conclusion: Our results have characterized the $\mathrm{m}^{6} \mathrm{~A}$-mRNA landscape in human HCC and revealed YTHDF2 as a molecular 'rheostat' in epitranscriptome and cancer progression.

Keywords: m $^{6}$ A, YTHDF2, HCC, Inflammation, Vessel normalization, IL-11, Serpin E2, HIF-2a antagonism

\section{Background}

As the most frequent internal decoration on eukaryotic mRNAs, $N^{6}$-methyladenosine $\left(\mathrm{m}^{6} \mathrm{~A}\right)$ has been proven to play fundamental roles in regulating gene expression and biological processes [1-3]. Pioneering studies have offered a glimpse into the sophisticated mechanisms of reversible $\mathrm{m}^{6} \mathrm{~A}$ modification in cancer. The demethylase FTO (fat

\footnotetext{
* Correspondence: houjj@sysucc.org.cn; chuanhe@uchicago.edu; xiaqiang@shsmu.edu.cn

${ }^{\dagger}$ Jiajie Hou, He Zhang and Jun Liu contributed equally to this work. 'Department of Liver Surgery, Renji Hospital, School of Medicine, Shanghai Jiaotong University, Shanghai 200127, China

${ }^{6}$ Department of Chemistry, Department of Biochemistry and Molecular Biology, Institute for Biophysical Dynamics, University of Chicago, Chicago, IL 60637, USA

Full list of author information is available at the end of the article
}

mass-and obesity-associated protein) decreases global $\mathrm{m}^{6} \mathrm{~A}$ levels, leading to either downregulation of tumor suppressor genes or upregulation of tumor promoter genes $[4,5]$. Another demethylase ALKBH5 (a-ketoglutarate-dependent dioxygenase alkB homolog 5) tends to stabilize stem-cellrelated transcripts $[6,7]$. METTL3 (methyltransferase-like 3)-catalyzed RNA methylation is required for cancer development [8-12], whereas the role of METTL14 varies with cancer types [13-16]. Diverse post-transcriptional outcomes of $\mathrm{m}^{6} \mathrm{~A}$-methylation rely on its readers, which are poorly understood in human cancers. YTHDF2 (YTH domain family 2) is the most effective $\mathrm{m}^{6} \mathrm{~A}$ reader that weakens mRNA stability by recognizing and distributing $\mathrm{m}^{6} \mathrm{~A}$-containing mRNAs to processing bodies $[17,18]$. Recent reports

(C) The Author(s). 2019 Open Access This article is distributed under the terms of the Creative Commons Attribution 4.0 International License (http://creativecommons.org/licenses/by/4.0/), which permits unrestricted use, distribution, and 
referred YTHDF2 was able to degrade both tumor promoter and suppressor gene mRNAs $[5,8,16,19,20]$. While it enhanced self-renewing of leukemic stem cells [20], the basic role of endogenous YTHDF2 in solid cancer remained in question.

Despite the identification of thousands of $\mathrm{m}^{6} \mathrm{~A}$ sites in a number of human cancer cell lines, less is known about the epigenomic alterations during cancer progression and the epitranscriptomic features amongst patients with any particular type of cancer. The metabolic traits of cancer cells, which are critical for adapting to the changes within the tumor microenvironment, often contribute to the epigenetic plasticity. As one of the most well-recognized cancer hallmarks, hypoxia can affect DNA methylation and histone methylation or acetylation by reducing enzymatic activity, regulating gene expression or increasing metabolite production $[21,22]$. In terms of $\mathrm{m}^{6} \mathrm{~A}-\mathrm{mRNA}$ editing, hypoxiainducible factors (HIFs) upregulate ALKBH5 expression, which in turn leads to NANOG mRNA demethylation in breast cancer cells [7]. Nonetheless, the oncometabolite 2hydroxyglutarate (2HG) attenuates FTO activity, thereby increasing global $\mathrm{m}^{6} \mathrm{~A}$ RNA modification in 2HG-sensitive leukemia or glioma cells $[5,23]$. Another study reported that hypoxia broadly increases $\mathrm{m}^{6} \mathrm{~A}$ modification to mRNAs, leading to the acquisition of higher mRNA stability instead of $\mathrm{m}^{6} \mathrm{~A}$-mediated decay [24]. These findings all suggest that tumor hypoxia causes $\mathrm{m}^{6} \mathrm{~A}$ epigenetic remodeling, but the prevalent alterations and the molecule which triggers these alterations have not yet been determined.

Hepatocellular carcinoma (HCC), which possesses the hallmarks of hypoxia and chronic inflammation, is one of the most lethal malignancies worldwide $[25,26]$. Here we identify a characteristic gain of $\mathrm{m}^{6} \mathrm{~A}$ modification in tandem with an increase of mRNA expression in both human HCC specimens and cell lines exposed to hypoxia. In an epitranscriptome-wide setting, these "hyperupregulated" genes exhibit a remarkable association with molecular rewiring for cancer progression. The reduction of YTHDF2, which predicts poor classification and prognosis of HCC patients, highly correlates with this epitranscriptional orchestration and promotes tumor growth and metastasis. Through genetic remodeling and $m^{6} A-m R N A$ profiling, we link YTHDF2 deficiency to mRNA stabilization of IL-11 and Serpin E2, the key mediators in support of hypoxia-induced cancer cell survival and vascular reconstruction [27, 28]. We further reveal a transcriptional inhibition of YTHDF2 by HIF$2 \alpha$, which has been therapeutically targeted in renal cell carcinoma and HCC $[29,30]$. Administration of a firstin-class HIF-2 $\alpha$ antagonist (PT2385) [31] restored and required YTHDF2 to suppress inflammation-associated cancer progression. Together, our data highlight a protective role of YTHDF2 in HCC interrupting the hypoxia-induced epigenetic-inflammation-cancer axis.

\section{Results}

Human HCC manifests a 'hyper-up' alteration in $\mathrm{m}^{6} \mathrm{~A}$ mRNA landscape

Despite the $\mathrm{m}^{6} \mathrm{~A}$ decrease in total RNA of human HCC, there is still no consensus about mRNA modification $[8,13]$. To assess the global $\mathrm{m}^{6} \mathrm{~A}$-methylation in HCC, we examined 37 tumor paired with adjacent non-tumor samples. By performing dot blot or liquid chromatography-tandem mass spectrometry (LC-MS/MS), we observed a slight $\mathrm{m}^{6} \mathrm{~A}$ decrease in total RNA, and yet a significant $\mathrm{m}^{6} \mathrm{~A}$ increase in mRNA, while comparing tumor tissues with autologous paratumor tissues (Additional file 1: Figure S1A, B and Fig. 1a). Next, eight pairs of mRNA samples were immunoprecipitated using an $\mathrm{m}^{6} \mathrm{~A}$-specific antibody in combination with high-throughput sequencing (MeRIP-seq). An average of 70.80 and $29.20 \% \mathrm{~m}^{6} \mathrm{~A}$ peaks showed a significant increase and decrease, respectively, in tumor relative to nontumor samples (Additional file 1: Figure S1C). Through analysis of RNA sequencing (RNA-seq) data, we noticed most transcripts that gained in $\mathrm{m}^{6} \mathrm{~A}$ manifested higher mRNA expression in tumor versus non-tumor counterparts. The superiority of 'hyper-up' population was found in 7 out of 8 patients, averagely taking up $69.27 \%$ amongst all the $\mathrm{m}^{6} \mathrm{~A}-\mathrm{la}$ belled transcripts (Fig. 1b and Additional file 1: Figure S1D, E). We then analyzed those high-frequency 'hyper-up' genes (at least shared by 3 patients), which are mainly responsible for cancer-promoting events, as indicated by Gene ontology (GO) analysis (Additional file 1: Figure S1F).

Using gene set enrichment analysis (GSEA), a previously established hypoxia signature [32] was strongly skewed toward tumor counterparts in the case of HCC (Fig. 1c). A recent study suggested hypoxia stimulated $\mathrm{m}^{6} \mathrm{~A}$ demethylation $^{6}$ whereas another report showed hypoxia favored $\mathrm{m}^{6} \mathrm{~A}$ labelling [24]. Therefore, we exposed HCC cell lines to hypoxia and observed a gain of $\mathrm{m}^{6} \mathrm{~A}$ in all four cell lines as compared to normoxic conditions (Fig. 1d). Using MeRIP-seq, we detected a larger number of $\mathrm{m}^{6} \mathrm{~A}$ peaks in hypoxic SMMC7721 mRNA, preferentially enriched in 3' untranslated regions (3'UTR) and coding regions (CDS) (Fig. 1e, f). As profiled by RNA-seq, transcripts gained in $\mathrm{m}^{6} \mathrm{~A}$ upon hypoxia manifested profoundly increased expression (Fig. 1g), which recapitulated the 'hyper-up' pattern identified in HCC specimens. GO analysis of those 'hyper-up' genes suggested activation of hypoxia-related oncogenic pathways (Additional file 1: Figure S1G). Notably, gene signatures of cell-cell contact and 'serine-type endopeptidase inhibitor activity' are enriched in 'hyper-up' populations based on both tumor tissue and hypoxic cell profiling (Additional file 1: Figure S1F, G).

\section{YTHDF2 is associated with HCC epitranscriptome and clinical outcome}

To analyze how hypoxia was linked to $\mathrm{m}^{6} \mathrm{~A}-\mathrm{mRNA}$ editing, we measured enzyme expression in HCC tissues as well as 


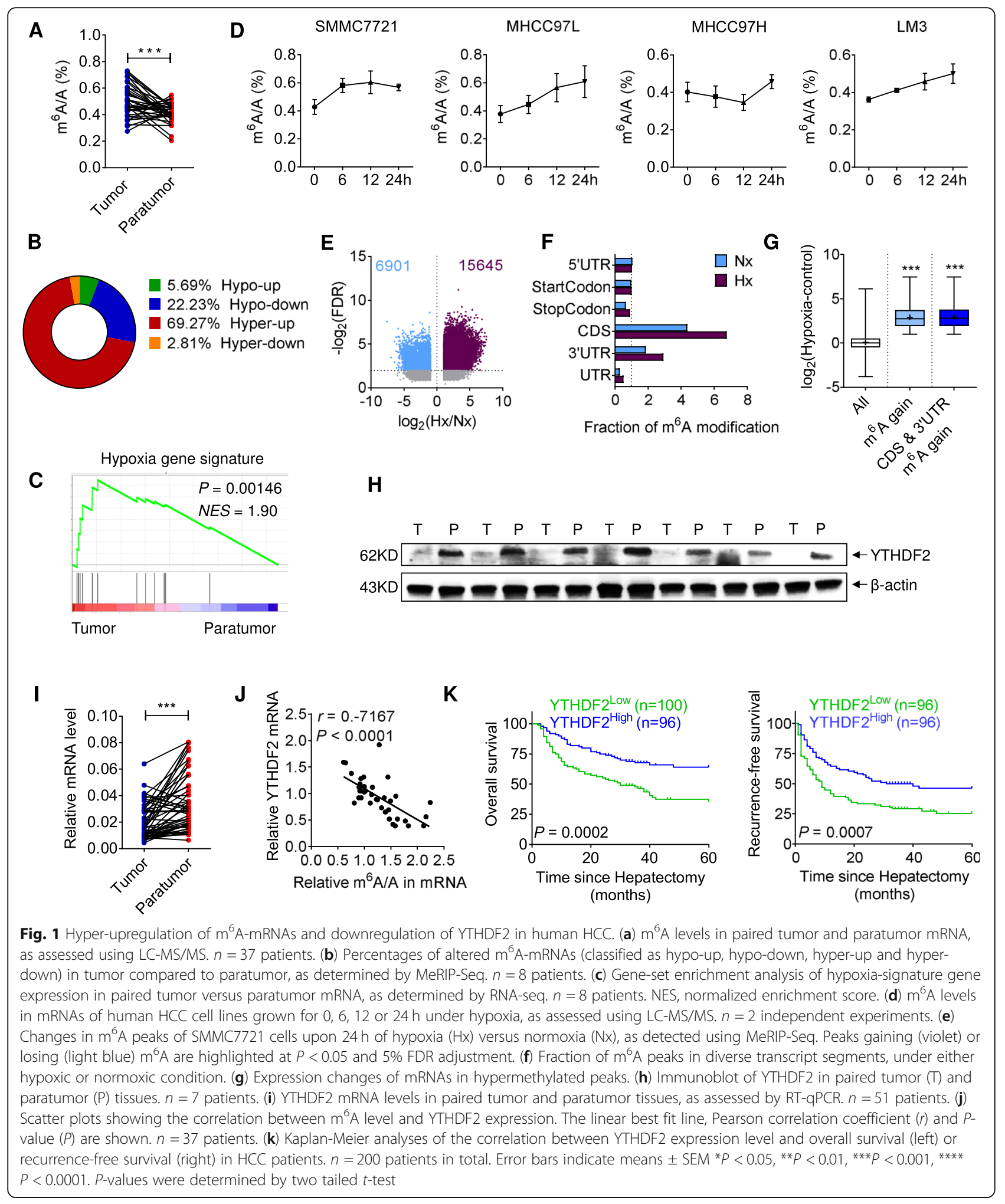

hypoxic cancer cells. Among methyltransferases, METTL14 mRNA displayed significant alteration in tumor (Additional file 1: Figure S2A), and yet its decrease could not explain the observed increase in global $\mathrm{m}^{6} \mathrm{~A}$. In concert with $\mathrm{m}^{6} \mathrm{~A}$ hypermethylation, FTO and ALKBH5 mRNAs decreased in tumor tissues; however, they both increased under hypoxic conditions (Additional file 1: Figure S2A, B). Notably, YTHDF2 mRNA was reduced in both hypoxic cells and 
tumor tissues, which indicated an inverse correlation with the m6A/A ratio (Additional file 1: Figure $\mathrm{S} 1 \mathrm{H}$ and $1 \mathrm{I}, \mathrm{J}$ ). Putatively, 'hyper-up' regulation of the $\mathrm{m}^{6} \mathrm{~A}$-epitranscriptome in $\mathrm{HCC}$ might result from the YTHDF2 reduction and consequential $\mathrm{m}^{6} \mathrm{~A}-\mathrm{mRNA}$ stabilization. We verified YTHDF2 protein levels were also decreased in hypoxic cells and tumor specimens (Additional file 1: Figure S1I, $1 \mathrm{H}$ and S2C). Importantly, in a setting of $200 \mathrm{HCC}$ patients (recruited from 2006 to 2012), lower YTHDF2 protein levels were significantly associated with more multinodular tumors and microvascular invasion, higher TNM and BCLC stage classification, and shorter overall and recurrence-free survival period (Additional file 2: Table S1, Additional file 1: Figure S2D and $1 \mathrm{~K})$. Accordingly, we hypothesized that a hypoxiasensitive YTHDF2 reduction reprogrammed the $\mathrm{m}^{6} \mathrm{~A}$-edited transcriptome and promoted HCC development.

\section{YTHDF2 deficiency promotes HCC growth, vasculature remodeling and metastasis}

A recent report has preliminarily showed that overexpression of YTHDF2 could suppress HCC growth [19], but the importance of endogenous YTHDF2 in HCC cells was still not clear. Hence, we stably silenced YTHDF2 expression in human HCC cell line SMMC7721 and MHCC97H (Additional file 1: Figure S3A, B), and assessed their biological behaviors. Disruption of YTHDF2 greatly benefited cell viability but did not retard apoptosis (Fig. 2a and Additional file 1: Figure S3C, D). Impressively, this acquired growth competence was conserved under hypoxic conditions (Fig. 2a). In terms of hypoxia-related events, we further evaluated cell migration, stemness, metabolism, and tumor angiogenesis [33]. YTHDF2 knockdown resulted in a promotion of angiogenic sprouting of human umbilical vein endothelial cells (HUVECs) in a co-culture system (Fig. 2b and Additional file 1: Figure S3E), but unaffected other cellular behaviors (Additional file 1: Fig. S3F-H). We next exploited a subcutaneous xenograft mouse model to monitor in vivo tumor development. YTHDF2 silencing robustly facilitated tumor growth and metastasis in NPG (NOD-Prkdc ${ }^{\text {scid }} I l 2 r g^{\text {null }}$ ) mice (Fig. 2c-e), and this was partially due to the effects on cell proliferation and tumor angiogenesis (Additional file 1: Figure S3I, $\mathrm{K}$ and Fig. 2f). It is well-documented that tumor vascular permeability and mimicry contributes to extravasation and metastasis [28, 34]. In line with this, YTHDF2 silencing in $\mathrm{MHCC} 97 \mathrm{H}$ tumors led to increased dextran leakage and $\mathrm{PAS}^{+} \mathrm{CD} 31^{-}$fluid-filled channels lined by tumor cells, indicating intensified vascular permeability and mimicry (Additional file 1: Figure S3J, L and Fig. 2g). In contrast, overexpression of YTHDF2 not only arrested cancer cell growth but also reduced vessel density and permeability (Additional file 1: Fig. S4A-H).

To obtain a deeper insight into the impact of YTHDF2 deficiency on HCC, we generated the liver-specific Ythdf2 KO mouse strain (Ythdf $2^{\mathrm{LKO}}$ ) and employed a chemical-induced HCC model. As compared with Ythdf2 $2^{\mathrm{F} / \mathrm{F}}$ littermates, Ythdf2 $2^{\mathrm{LKO}}$ mice developed more advanced liver lesions, in terms of macroscopic number, size and histology, and more lung metastases (Fig. 2h-j and Additional file 1: Figure S4I). Tumor proliferation, apoptosis and angiogenesis were enhanced in the YTHDF2-deficient mouse liver (Additional file 1: Figure S4J, $\mathrm{K}$ and Fig. 2k). Nonetheless, no obvious vascular mimicry existed in either $Y$ thdf $2^{\mathrm{F} / \mathrm{F}}$ or $Y$ thdf $2^{\mathrm{LKO}}$ liver tumors (data not shown). To our knowledge, the microvascular network in solid tumors is often compromised with functional abnormalities, which fuel metastasis formation [35]. In the absence of YTHDF2, NG2 ${ }^{+}$pericytes were significantly reduced, whereas $\mathrm{CD}^{+} 1^{+}$endothelial cells largely accumulated and formed microvessels in tumor regions (Fig. 2k), defining a fundamental role for YTHDF2 in vascular normalization [35].

\section{YTHDF2 deficiency escalates inflammation and vessel abnormalization}

We subsequently performed RNA-seq in control and YTHDF2-deficient SMMC-7721 cells. Taking into consideration that putative targets were retarded for degradation, we mainly focused on the upregulated transcripts (Fig. 3a). GO analysis revealed an enrichment of a cancerpromoting inflammation program, as characterized by the top GO term 'secretion by cell' and 'positive regulation of tyrosine phosphorylation of Stat3 protein' (Fig. 3b and Additional file 3: Table S2). We did not see epidermal growth factor receptor (EGFR) among the upregulated genes, although it has been recently reported elsewhere [19]. To find out the key determinants in a hypoxic cancer context, we used a combined screening approach by integrating YTHDF2-regulated transcriptional profiling and the hypoxia-responsive epitranscriptome. Therefore, we selected 182 genes that were increased by over 2-fold upon YTHDF2 knockdown, and 1122 hypermethylated transcripts that were upregulated by over 2-fold under hypoxic conditions (Fig. 3c). Precisely, these genes intersected for 21 candidate targets, among which IL11 and SERPINE2 were most relevant to cancer-promoting inflammation. IL-11 promotes STAT3 activation and inflammatory cancer progression in an autocrine manner [27, 36], while cancer cell-secreted serpin E2 confers invasive and metastatic processes by reprogramming the tumor vasculature [28, 37-39]. We validated that both STAT3 phosphorylation and IL11 and SERPINE2 expression were upregulated in YTHDF2-deficient cells, and this effect could be reinforced by oxygen deficit (Fig. 3d-g and 


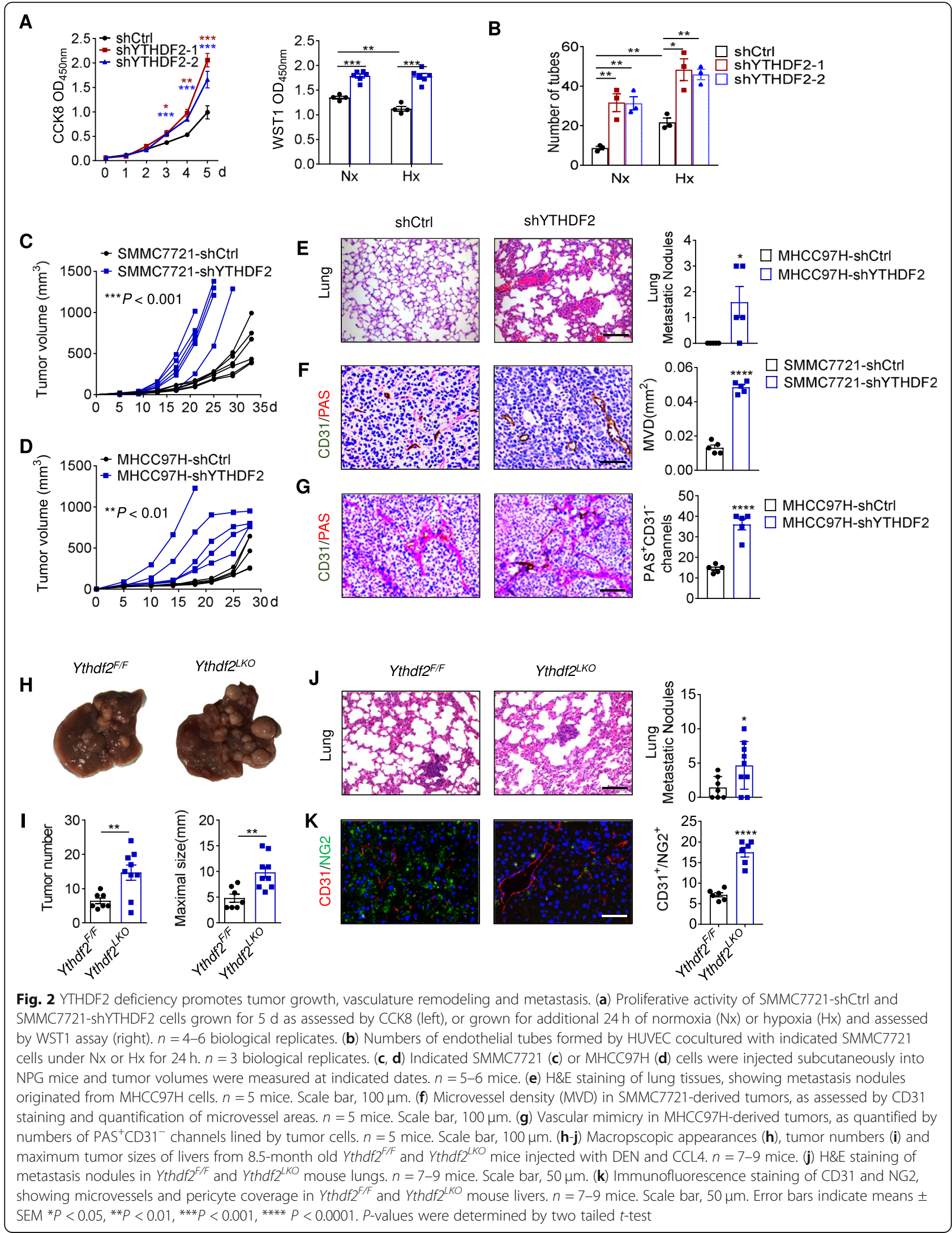


A

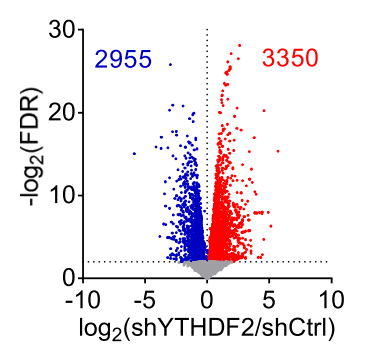

C

YTHDF2 knockdown Hypoxia

Upregulated genes "Hyper-up" genes

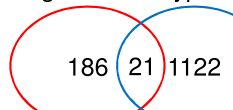

SERPINE2, ITGA5, IL11, MFGE8, CA12,

SHANK3, TNNT1, MX11, GRB10, PCDH1,

N4BP3, ARID5A, FAM171A2, SDR16C5, NEU1,

TTYH3, CXCR4, ID1, DUSP10, JUNB, PPP2R5B

E

IL11
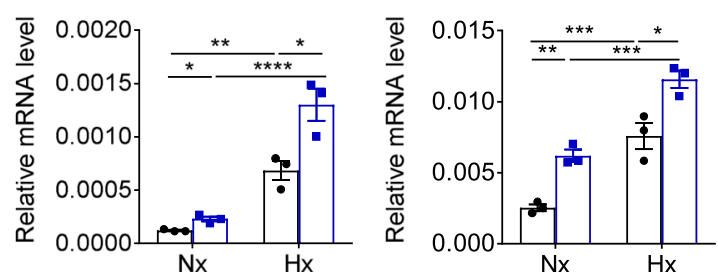

G

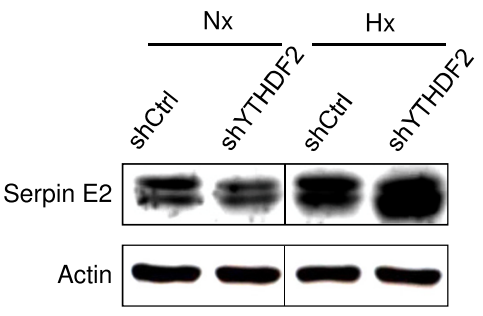

I

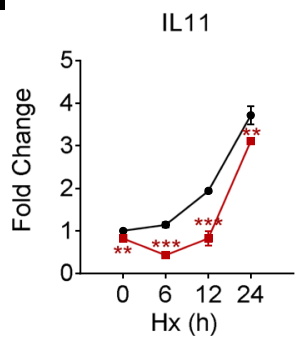

SERPINE2

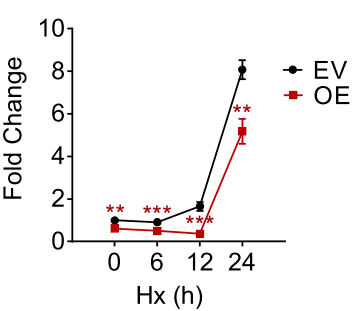

GO terms

Secretion by cell-

$-\log _{10}(p$ value $)$

Cellular response to peptide-

Cytokine-mediated signaling pathway-

Inflammatory response
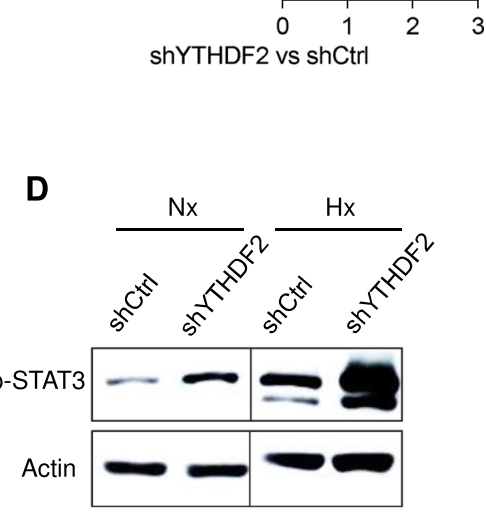

$\mathbf{F}$

IL-11

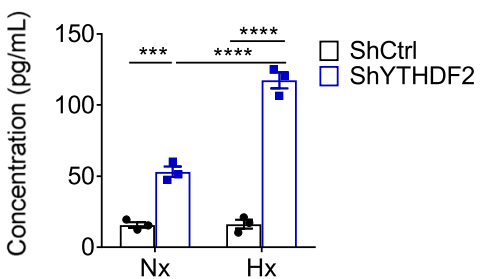

H

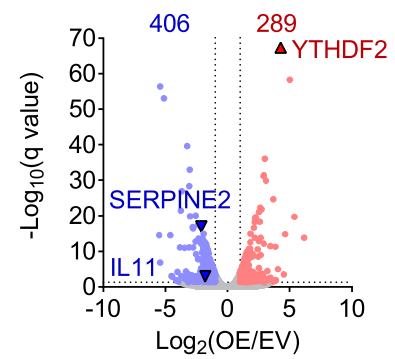

J

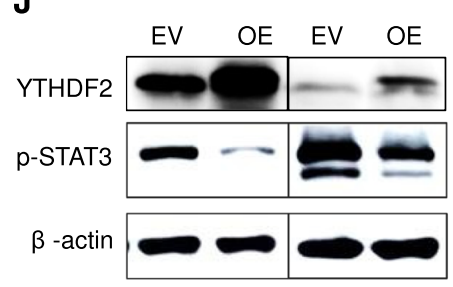

Fig. 3 (See legend on next page.) 


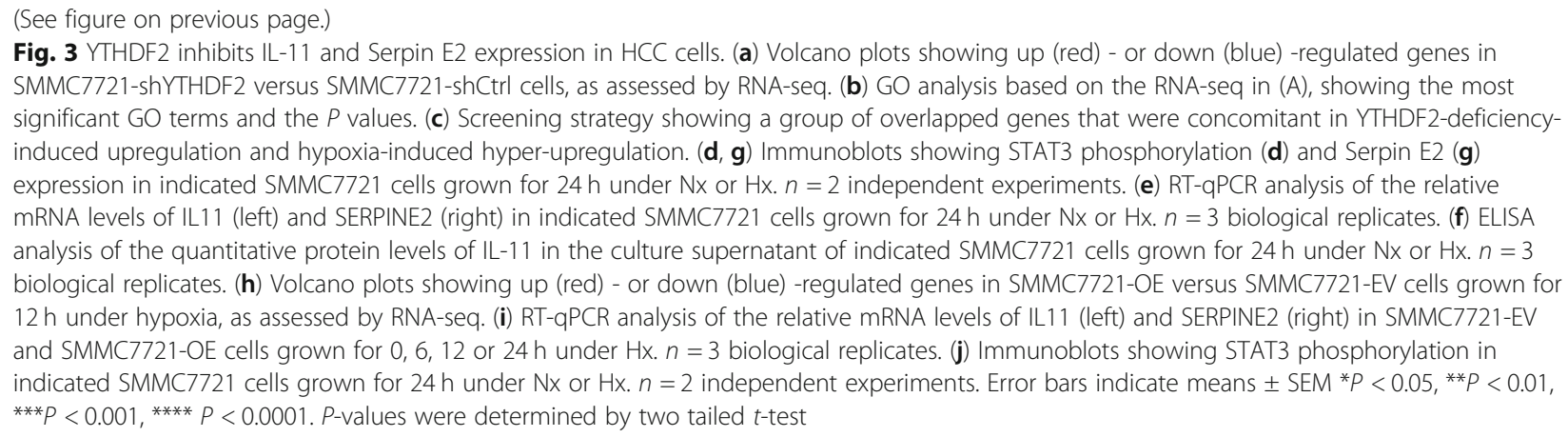

Additional file 1: Figure S5A-D). In parallel, YTHDF2 deficiency in mouse hepatocytes yielded IL11 and SERPINE2 expression (Additional file 1: Fig. S5E). Conversely, RNA profiling and qPCR detection of YTHDF2-overexpressed cells showed substantial downregulation of IL11 and SERPINE2, as well as phosphorylation of STAT3, in comparison to the control cells (Fig. 3i-j and Additional file 4: Table S3). Unexpectedly, short-term (12 h) exposure to hypoxia unleashed the repressive properties of YTHDF2 toward IL11 and SERPINE2 mRNAs (Fig. 3I).

\section{YTHDF2 degrades $\mathrm{m}^{6} \mathrm{~A}$-containing IL11 and SERPINE2 mRNAs}

Using $\mathrm{m}^{6} \mathrm{~A}$-specific $\mathrm{qPCR}$, we uncovered that YTHDF2 induced $\mathrm{m}^{6} \mathrm{~A}$ loss in IL11 and SERPINE2 transcripts (Fig. 4a). To investigate whether YTHDF2 directly recognized $\mathrm{m}^{6} \mathrm{~A}$ marked IL11 and SERPINE2 mRNAs, we performed mRNA-immunoprecipitation (RIP) for YTHDF2. Subsequent RT-qPCR identified IL11 and SERPINE2 as YTHDF2 substrates, particularly under hypoxic condition (Fig. 4b). We then asked whether YTHDF2 processes IL11 and SERPINE2 mRNAs to decay. After actinomycin D treatment, their lifetimes were prolonged in YTHDF2-silenced cells and were shortened in YTHDF2-overexpressed cells (Fig. 4c, d). Although hypoxia dramatically delayed IL11 and SERPINE2 degradation in control cells, YTHDF2 overexpression expedited the processing to a normal level. Since human HCC tissues exhibited high abundance of $\mathrm{m}^{6} \mathrm{~A}$ modification to the consensus sites of IL11 and SERPINE2 3'UTRs (Fig. 4e), we inserted their transcript segments harboring $\mathrm{m}^{6} \mathrm{~A}$ motifs into a pmirGLO reporter plasmid [17]. Under hypoxic conditions, introduction of exogenous YTHDF2 efficiently disrupted reporter activity in the presence of $\mathrm{m}^{6} \mathrm{~A}$ motifs (Fig. 4f). Using fluorescence in situ hybridization (FISH), we visualized localization of YTHDF2 and target mRNAs to processing bodies (Fig. 4g, h). As expected, YTHDF2 affected both expression and distribution of IL11 and SERPINE2 mRNAs. Noticeably, $12 \mathrm{~h}$ of exposure to hypoxia induced YTHDF2 translocation to processing bodies, substantially supporting its provisional potency propelled by a lack of oxygen (Fig. 4g).
As a specific $\mathrm{m}^{6} \mathrm{~A}$ 'reader' protein, YTHDF2 depends on the hydrophobic residues W432 and W486 in its carboxy-terminal YTH domain for selective recognition of $\mathrm{m}^{6} \mathrm{~A}[17,40]$. To understand the molecular basis of our findings, we mutated the $\mathrm{m}^{6} \mathrm{~A}$ recognition sites into W432A and W486A, which abrogate the specific binding affinity of YTHDF2 (Additional file 1: Figure S6A, B). A technical deviation is that the strain carrying a W432A mutation showed a little bit lower YTHDF2 protein level than wild-type control, but the W486A version was comparable to wild-type. In YTHDF2-deficient cells, re-expression of wild-type YTHDF2 but not the catalytically inactive mutant offset the acquired cancerpromoting inflammatory phenotypes (Fig. 5a, b), and reduced both phosphorylation of STAT3 and stabilization of IL11 and SERPINE2 mRNAs (Fig. 5ce and Additional file 1: Figure S6C, D). In concert with this, redundant expression of catalytically dead YTHDF2 failed to inhibit in vivo tumor development (Fig. $5 \mathrm{f}$ and Additional file 1: Figure S6E). These results suggest that the $\mathrm{m}^{6} \mathrm{~A}$ reader function is indispensable for the suppressive role of YTHDF2 in HCC.

To ascertain the importance of YTHDF2-IL11/SERPINE2 pathway in HCC development, we silenced IL11 or SERPINE2 in YTHDF2-deficient cells by using shRNAs. Removal of IL11 abrogated the growth competitiveness of YTHDF2-deficient SMMC7721 cells (Fig. 5g), while a SERPINE2 deficit attenuated the proangiogenic capacity of SMMC7721 cells (Fig. 5h), highlighting that IL11 and SERPINE2 are key targets of YTHDF2 in cancer-promoting inflammation. As anticipated, concomitant targeting of both IL11 and SERPINE2 counterbalanced the malignant capability endowed by YTHDF2 depletion (Fig. 5i and Additional file 1: Figure S6F). To examine this pathway in a clinical setting, we looked into human HCC tissues with relatively low or high YTHDF2 protein levels. As shown in Fig. 5j, IL-11 and Serpin E2 expression displayed negative correlations with YTHDF2. 


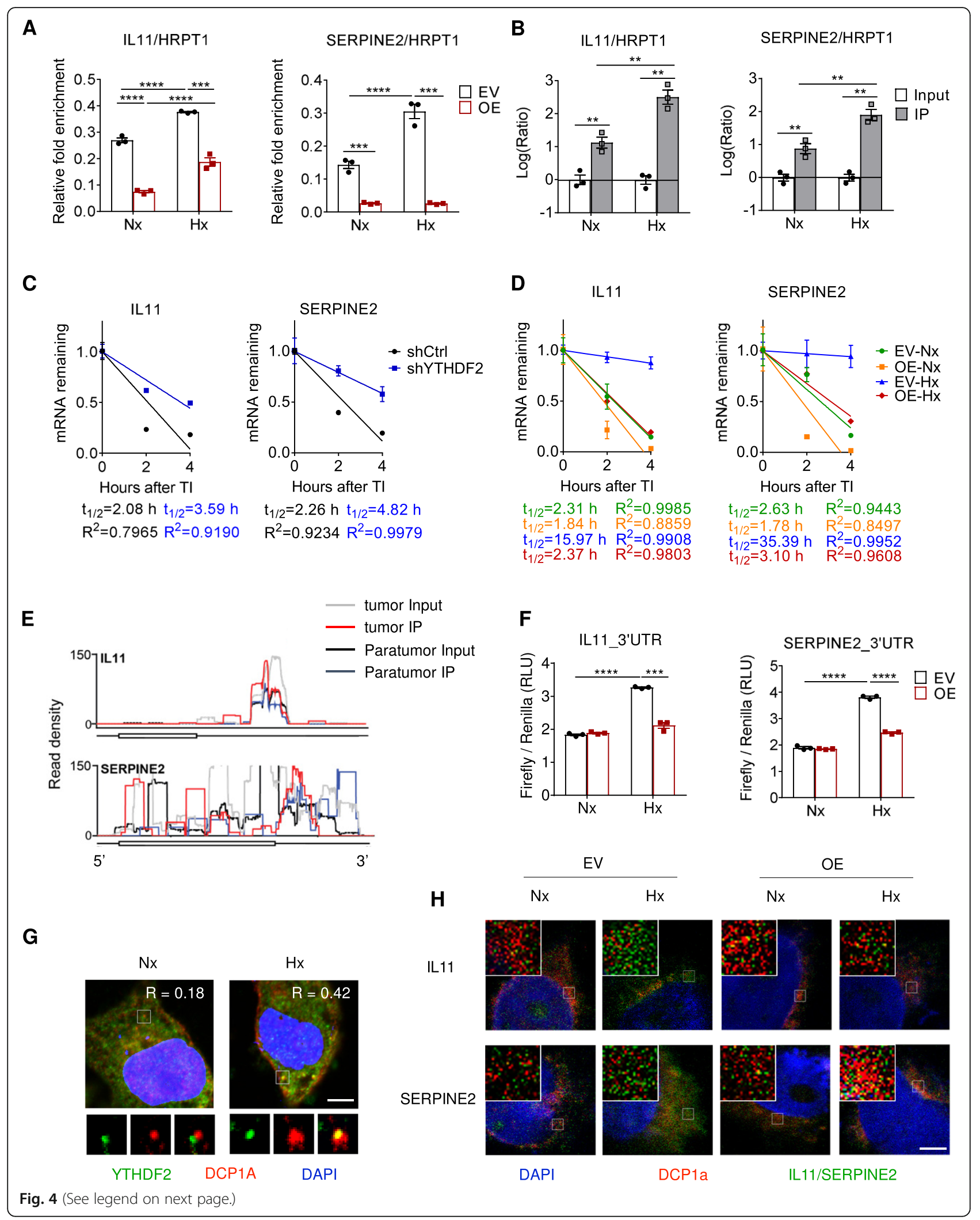


(See figure on previous page.)

Fig. 4 YTHDF2 processes $m^{6} A$-marked IL11 and SERPINE2 mRNAs for decay. (a) $m^{6} A$-enrichment in the IL11 and SERPINE2 mRNAs in SMMC7721 cells expressing empty vector (EV) or overexpressing YTHDF2 (OE), as assessed by MeRIP-qPCR. The cells were subjected to Nx or Hx for $12 \mathrm{~h}$. $n=$ 3 biological replicates. (b) Gene enrichment of IL11 and SERPINE2 in YTHDF2-mRNA complex immunoprecipitated from SMMC7721-OE cells, as determined by RIP-qPCR. The cells were subjected to Nx or Hx for $12 \mathrm{~h} . n=3$ biological replicates. (c, d) RNA lifetime of IL11 and SERPINE2 in indicated SMMC7721 cells, as determined by monitoring transcript abundance after transcription inhibition (TI). The cells were subjected to Nx or $\mathrm{Hx} 8 \mathrm{~h}$ prior to TI initiation. $n=3$ biological replicates. (e) The average read density showing the $\mathrm{m}^{6} \mathrm{~A}$ peaks identified in IL 11 and SERPINE2 transcripts in human HCC tissues as assessed using MeRIP-seq. $n=8$ patients. (f) Luciferase reporter assay showing posttranscriptional regulation by YTHDF2 in the presence of IL11 (left) or SERPINE2 (right) 3'UTR. Indicated SMMC7721 cells were grown for $12 \mathrm{~h}$ under $\mathrm{Nx}$ or Hx. Renilla luciferase activity was normalized to firefly activity and presented as relative luciferase activity. $n=3$ biological replicates. (g) Immunofluorescence staining of YTHDF2 and DCP1a (P-body marker) in YTHDF2-overexpressing SMMC7721 cells grown for $12 \mathrm{~h}$ under Nx or Hx. $n=3$ biological replicates. Scale bar, $5 \mu \mathrm{m}$. (h) Fluorescence in situ hybridization of IL11 or SERPINE2 mRNA and immunofluorescence staining of DCP1a in indicated SMMC7721 cells grown for $12 \mathrm{~h}$ under Nx or Hx. $n=3$ biological replicates. Scale bar, $5 \mu \mathrm{m}$. Error bars indicate means \pm SEM $* P<0.05$, ${ }^{* *} P<0.01,{ }^{* *} P<0.001,{ }^{* * *} P<0.0001$. $P$-values were determined by two tailed $t$-test

\section{Hypoxia interrupts YTHDF2 expression in a HIF-2a- dependent manner}

Hypoxic tumor areas showed relatively low YTHDF2 expression in the xenograft mouse model (Fig. 6a), providing an in vivo evidence for hypoxia-mediated YTHDF2 reduction. A recent report showed that HIF-1 $\alpha$ was responsible for this negative regulation [19]. However, when we sought to block the hypoxia signaling in vitro, only targeting HIF- $2 \alpha$ completely rescued YTHDF2 expression in hypoxic cells (Additional file 1: Figure S7A-C and Fig. 6b, c). Containing the hypoxia-responsive elements (HREs, CGTG), the Ythdf2 promoter was assessed through chromatin immunoprecipitation (ChIP)-qPCR and demonstrated HIF-2 $\alpha$ binding (Fig. 6d). Additionally, we inserted the Ythdf2 promoter into a pGL4 luciferase reporter plasmid. While Ythdf2 promoter activity was decreased upon hypoxia, this was reversed by a HIF- $2 \alpha$ (but not HIF-1 $\alpha$ ) siRNA (Fig. 6e and Additional file 1: Figure S7D). In human tissues, HIF- $2 \alpha$ was detected in the cytoplasm of non-tumor hepatocytes (Additional file 1: Fig. S7E), whereas it was aggregated in the nucleus of tumor cells, where it could regulate gene transcription.

Since HIF-2 $\alpha$ often terminates further expansion of hypoxic tumors, a small molecule (PT2385) selectively targeting HIF- $2 \alpha$ has been developed to treat renal cell carcinoma and hepatocellular carcinoma [29, 30]. We tested PT2385 in treating HCC cells in vitro and in vivo, and found it exhibited favorable therapeutic effects (Fig. 6f, g, k). PT2385 rescued YTHDF2 expression (Fig. 6h) but unaffected YTHDF2 localization in hypoxic cells (data not shown), accompanied by neutralization of IL11 and SERPINE2 expression, as well as STAT3 phosphorylation (Fig. 6i, j). Nonetheless, PT2385 administration to the mice bearing YTHDF2-deficient tumors failed therapeutically (Fig. 6l), which was indicative of an indispensable role of YTHDF2 in treating hypoxic HCC.

\section{Discussion}

This study specified the suppressive potential of YTHDF2 in cancer-promoting inflammation, and established a context of metabolic-epigenetic regulation where hypoxia imposes a cancer-specific procedure of $\mathrm{m}^{6} \mathrm{~A}-\mathrm{mRNA}$ editing. Importantly, restoring YTHDF2 expression defined $\mathrm{m}^{6} \mathrm{~A}$ decoration as a tumor suppressive mechanism. Despite the sensitivity of destabilizing IL11 and SERPINE2 mRNAs in response to tumor hypoxia, YTHDF2 expression is readily silenced in the presence of active HIF-2 $\alpha$ (Fig. 7). In accordance, HIF- $2 \alpha$ blockade improves YTHDF2-mediated suppression on $\mathrm{m}^{6} \mathrm{~A}-\mathrm{mRNA}$ stability and inflammatory cancer behaviors, leveraging this metabolic-epigenetic axis toward therapeutic opportunities in human HCC.

Unlike methylation-related inhibition of tumor suppressor gene promoters, the epigenomic hallmarks and functional relevance for $\mathrm{m}^{6} \mathrm{~A}$ mRNA decoration have not been fundamentally defined in human cancers. Here and elsewhere [13] it has been shown that $\mathrm{m}^{6} \mathrm{~A}$ labeling is blunted in total RNAs of human HCC tissues, and yet tumor mRNAs exhibit $\mathrm{m}^{6} \mathrm{~A}$ hypermethylation. This could be explained by the possibility of $\mathrm{m}^{6} \mathrm{~A}$ hypomethylation in other RNAs, since $\mathrm{m}^{6} \mathrm{~A}$ was also found in transfer RNA (tRNA), ribosomal RNA (rRNA), small nuclear RNA (snRNA) and long non-coding RNA (lncRNA) [41]. Here we attach importance to abnormal mRNA methylation-related gene expression and biological functions in human HCC. To map its epitranscriptomic landscape, we took advantage of $\mathrm{m}^{6} \mathrm{~A}$ - and mRNA-seq methods and thus quantitatively compared transcriptome-wide changes between tumor and nontumor tissues. Typically, the majority of $\mathrm{m}^{6} \mathrm{~A}$-labelled mRNAs were regulated in a 'hyper-up' manner, which assembled a variety of oncogenic pathways. Interestingly, introducing HCC cells to hypoxia enabled this epitranscriptomic transformation in vitro, affecting common oncogenes such as those involved in 'serine-type endopeptidase inhibitor activity'. Putatively, the 'hyper-up' feature is caused, at least in part, by hypoxia-induced YTHDF2 reduction. It has been widely accepted that steady-state levels of most transcripts decrease in the presence of $\mathrm{m}^{6} \mathrm{~A}$ modification. Nonetheless, a deficit in YTHDF2 expression may shift $\mathrm{m}^{6} \mathrm{~A}$-editing into a 
A

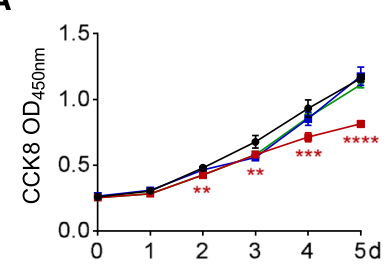

C
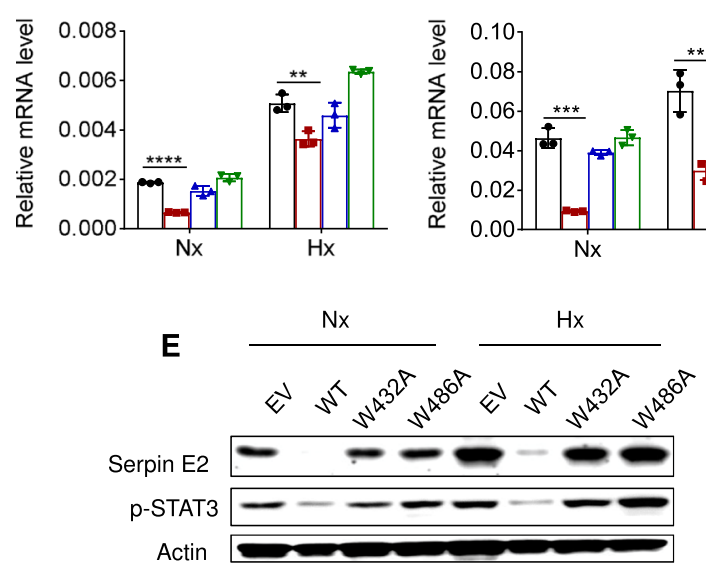

G

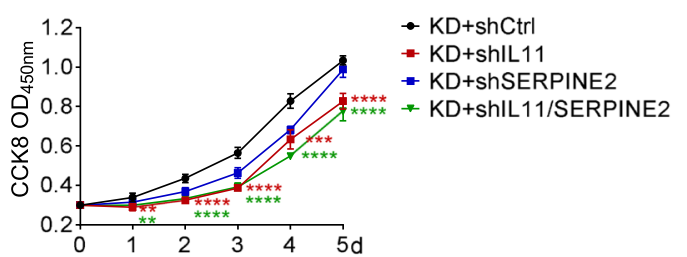

B
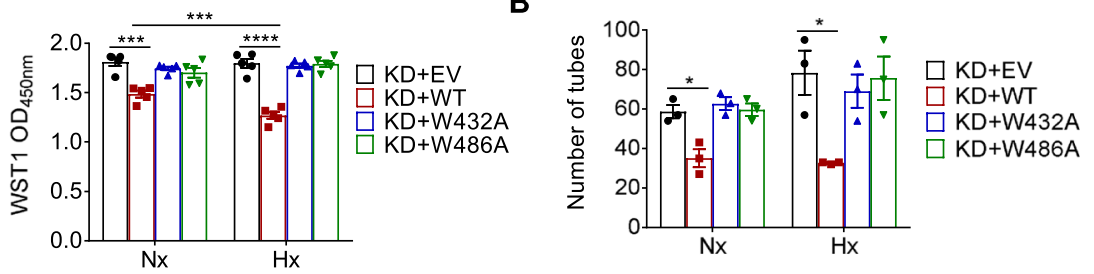

D
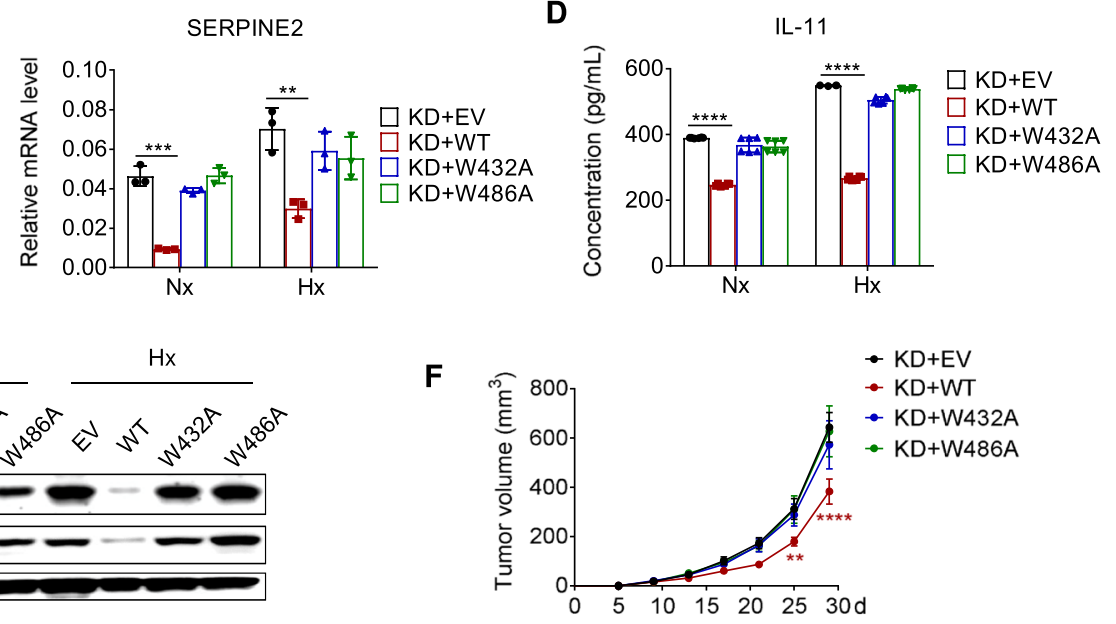

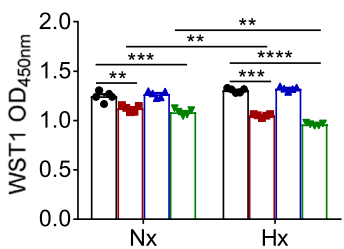

J YTHDF2 Low

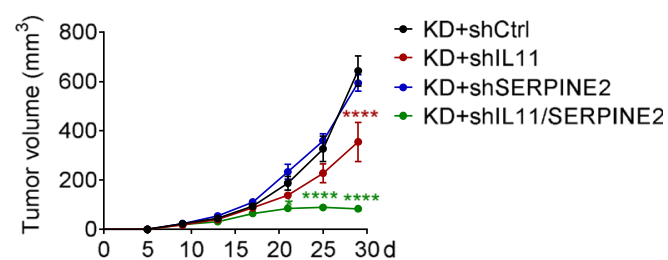

H

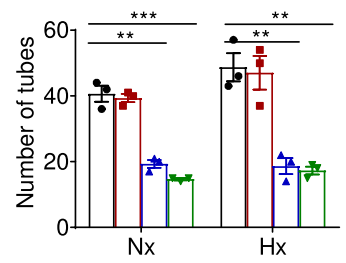

SERPINE2

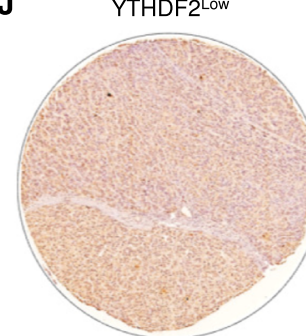

YTHDF2High

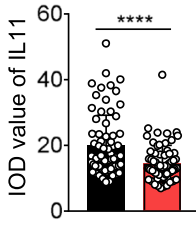

Fig. 5 (See legend on next page.) 


\begin{abstract}
(See figure on previous page.)
Fig. 5 YTHDF2 suppresses HCC by targeting IL11 and SERPINE2 mRNAs in a $m^{6} A$-reader fashion. (a) An empty vector (EV) or vectors encoding wild-type (WT) or mutant YTHDF2 (W432A and W486A) were transduced into SMMC7721 cells with a YTHDF2-knockdown (KD) background. Proliferative activity of indicated SMMC7721 cells was assessed by CCK8 (left) or WST1 assay (right). $n=5$ biological replicates. (b) Numbers of endothelial tubes formed by HUVEC cocultured with indicated SMMC7721 cells grown for $24 \mathrm{~h}$ under Nx or Hx. $n=3$ biological replicates. (c) RTqPCR analysis of the relative mRNA levels of IL11 (left) and SERPINE2 (right) in indicated SMMC7721 cells grown for $24 \mathrm{~h}$ under Nx or Hx. $n=3$ biological replicates. (d) ELISA analysis of the quantitative protein levels of IL-11 in the culture supernatant in indicated SMMC7721 cells grown for $24 \mathrm{~h}$ under Nx or Hx. $n=3$ biological replicates. (e) Immunoblots showing STAT3 phosphorylation and Serpin E2 expression in indicated SMMC7721 cells grown for $24 \mathrm{~h}$ under Nx or Hx. $n=2$ independent experiments. (f) Indicated SMMC7721 cells were injected subcutaneously into NPG mice and tumor volumes were measured at indicated dates. $n=5$ mice. (g) Control shRNA or shRNAs targeting IL11 and SERPINE2 were transduced into SMMC7721 cells with a YTHDF2-knockdown (KD) background. Proliferative activity of indicated SMMC7721 cells was assessed by CCK8 (upper) or WST1 assay (lower). $n=5$ biological replicates. (h) Numbers of endothelial tubes formed by HUVEC cocultured with indicated SMMC7721 cells. $n=3$ biological replicates. (i) Indicated SMMC7721 cells were injected subcutaneously into NPG mice and tumor volumes were measured at indicated time points. $n=5$ mice. (j) Immunohistological staining of IL-11 and SerpinE2 in human HCC tissue arrays divided as YTHDF2 $^{\text {Low }}$ and YTHDF2 ${ }^{\text {High }}$ categories according to median integrated optical density (IOD) value of YTHDF2. $n=143$ patients. Scale bar, $200 \mu \mathrm{m}$. Error bars indicate means \pm SEM ${ }^{*} P<0.05,{ }^{* *} P<0.01,{ }^{* *} P<0.001,{ }^{* * *} P<0.0001$. $P$-values were determined by two tailed $t$-test
\end{abstract}

cancer-specific mode. This notion is also supported by the recent observations that certain $\mathrm{m}^{6} \mathrm{~A}$-labelled genes become more stable in cancers [4, 42]. Taken together, our findings suggest hypoxia acts to shape the distinct $\mathrm{m}^{6} \mathrm{~A}-\mathrm{mRNA}$ landscape in human HCC.

Most HCC patients share a non-resolved inflammatory condition, owing to a history of chronic liver diseases with viral, fatty or other causes. In an autocrine or paracrine fashion, HCC cells receive the malignant signals from pro-inflammatory cytokines, which boost tumor and vascular proliferation and metastatic invasion. Blockade of oncogenic and angiogenic kinases or downstream STAT3 can retard primary tumor growth, which provides a rationale for many mainstream clinical therapeutics [43]. Here we identify YTHDF2 as a novel tumor suppressor resolving cancer-promoting inflammation. Mechanistically, YTHDF2 inhibited STAT3 phosphorylation and tumor growth by degrading IL11 mRNA, which encodes the dominant IL-6 family cytokine that endows gastrointestinal cancers with proliferative and invasive capacity $[27,36]$. The recent report showed that YTHDF2 could suppress EGFR phosphorylation [19], suggesting the substrate of YTHDF2 is in the upstream of EGFR but not EGFR itself.

Given the hypervascularity of HCC or metastatic liver cancer, anti-angiogenic therapy has long been considered unique; however, accumulating evidence in other cancer types have yielded insights into the necessity of both angiogenic and nonangiogenic mechanisms for targeting tumor vasculature. The latter accounts for the acquired resistance of antiangiogenic therapy and metastatic dissemination of cancer cells, including but not limited to vascular cooption and mimicry $[27,44]$. In this context, we unexpectedly found that YTHDF2 resisted either tumor angiogenesis or vascular mimicry by targeting SERPINE2. Serpins, or serine-type endopeptidase inhibitors, are a superfamily of secretory proteins acting as anti-coagulant and pro-inflammatory factors, which frequently display $\mathrm{m}^{6} \mathrm{~A}-\mathrm{mRNA}$ hypermethylation and gene overexpression in human HCC. Plasminogen activator (PA) inhibitory serpins have been shown to promote tumor growth and initiate metastasis by fostering angiogenesis and vascular co-option [37, 38]. As previously reported, the autocrine of serpin E2 of breast cancer cells enabled vascular mimicry and drove metastatic extravasation [28]. We show that YTHDF2 is required for suppressing tumor angiogenesis in vitro and in vivo, and restricting formation of vascular mimicry in the metastatic $\mathrm{MHCC} 97 \mathrm{H}$ tumors. Strikingly, YTHDF2 also benefited tumor vessel normalization [35], as characterized by a positive correlation to pericyte coverage and an inverse correlation to vascular permeability. Therefore, these results indicated that YTHDF2 provided the ideal molecular basis to control $\mathrm{HCC}$ by pleiotropic targeting of cancer-promoting inflammation.

Under normoxia, introduction of exogenous YTHDF2 in HCC cells could slightly mediate IL11 and SERPINE2 mRNA decay but did not correspondently disrupt the 3'UTR reporter activity in the presence of $\mathrm{m}^{6} \mathrm{~A}$ motifs. A possible explanation is that the simplified 3'UTR constructs could not faithfully reflect the interaction between substrate mRNAs and YTHDF2. By contrast, endogenous YTHDF2 seemed more functional than the exogenous version, hence the expression of IL11 and SERPINE2 mRNAs were significantly upregulated when knocking down YTHDF2. Importantly, YTHDF2 function was reinforced by oxygen deficit, owing to its increased distribution in processing bodies.

Hypoxia is a key driver of tumor growth, angiogenesis and metastasis, and also a mediator preconditioning revascularization and tumor dissemination post therapeutic vascular regression $[34,44]$. Clinically, hypoxia-induced HIF stabilization was associated with poor patient survival in a variety of cancer types [34]. Despite a small portion of overlapping target genes, HIF- $1 \alpha$ and HIF- $2 \alpha$ often exert different functions at different stage of cancer development. For 


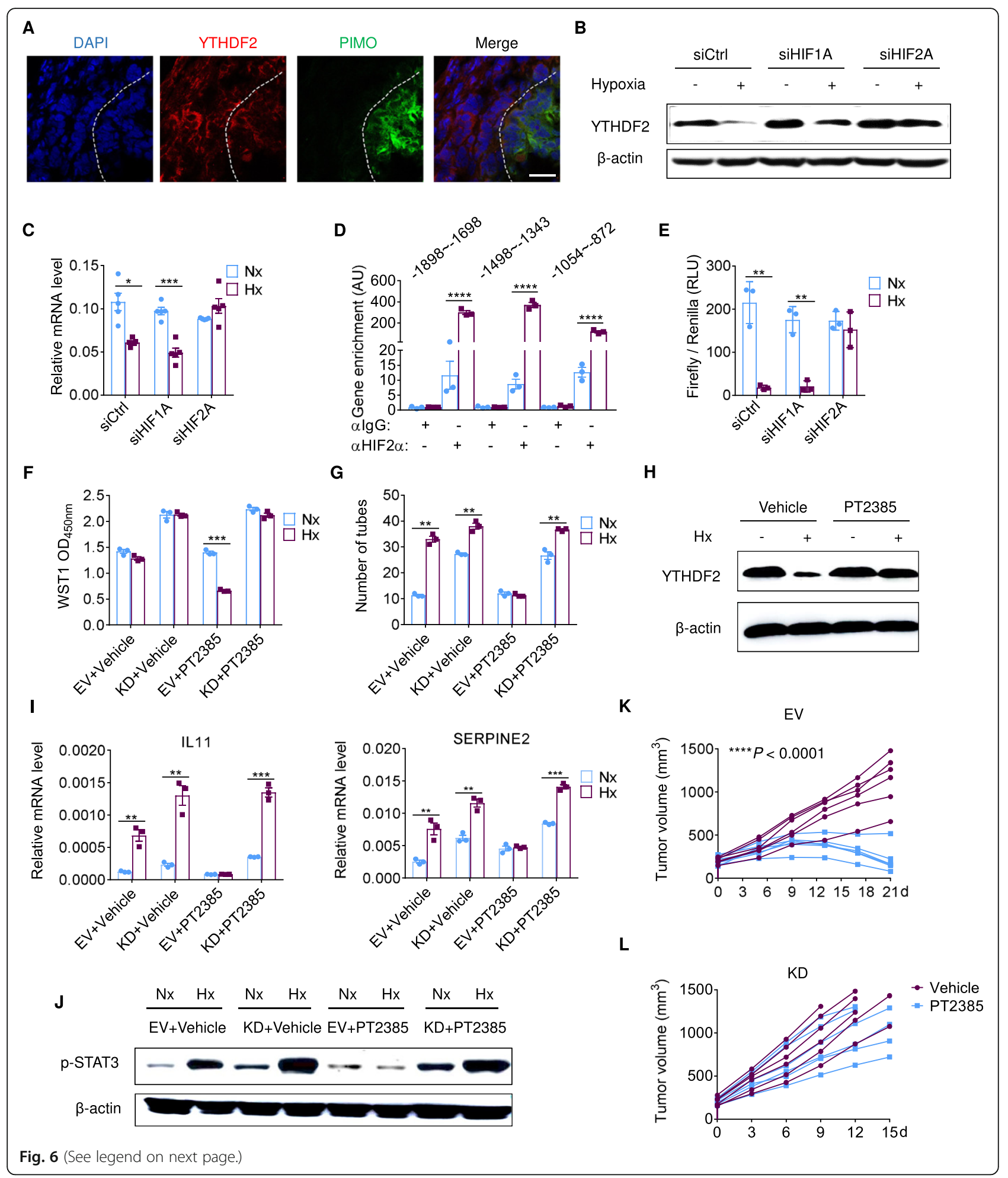


(See figure on previous page.)

Fig. 6 HIF-2a blockade contributed to YTHDF2-dependet HCC inhibition. (a) Immunofluorescence staining of YTHDF2 and pimonidazole (PIMO) in SMMC7721-derived mouse tumors. Hypoxic tumor areas were marked by PIMO staining. $n=3$ biological replicates. Scale bar, $20 \mu \mathrm{m}$.(b) Immunoblot of YTHDF2 in SMMC7721 cells transduced with a control siRNA (siCtrl) or siRNAs targeting HIF-1/2a and grown for $24 \mathrm{~h}$ under Nx or Hx. $n=2$ independent experiments. (c) RT-qPCR analysis of YTHDF2 in SMMC7721 cells expressing indicated siRNAs after $24 \mathrm{~h}$ of Nx or Hx exposure. (d) Enrichment of hypoxia-responsive elements (HREs) -containing fragments of human Ythdf2 promoter in DNA-HIF-2a complex, as determined by ChIP-qPCR. $n=4$ biological replicates. (e) Ythdf2 promoter activity in SMMC7721 cells expressing indicated siRNAs, as quantified using luciferase assay. Renilla luciferase activity was normalized to firefly activity and presented as relative luciferase activity. $n=3$ biological

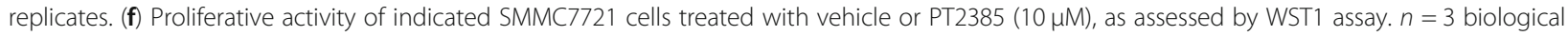
replicates. (g) Numbers of endothelial tubes formed by HUVEC cocultured with indicated conditions. $n=3$ biological replicates. (h, j) Immunoblot of YTHDF2 (h) and p-STAT3 (j) in SMMC7721 cells treated with vehicle or PT2385 (10 $\mu \mathrm{M}) . n=2$ independent experiments. (i) RT-qPCR analysis of IL11 (left) and SERPINE2 (right) mRNA levels. $n=3$ biological replicates. (k, I) NPG mice bearing SMMC7721-shCtrl (K) or SMMC7721-shYTHDF2 (L) cells were orally treated with Vehicle or PT2385 $(20 \mathrm{mg} / \mathrm{kg} / \mathrm{d})$ after reaching an average tumor volume of $200 \mathrm{~mm}^{3}$. Tumor volumes were continuously measured at indicated dates of treatment. $n=6$ mice. Error bars indicate means \pm SEM ${ }^{*} P<0.05,{ }^{* *} P<0.01,{ }^{* * *} P<0.001$, ${ }^{* * * *} P<$ 0.0001. P-values were determined by two tailed t-test

instance, HIF- $1 \alpha$ and HIF- $2 \alpha$ play complementary roles during early angiogenesis, whereas vascular remodeling are mainly HIF-2 $\alpha$-driven [45]. In certain types of cancer, a HIF-1 $\alpha$-to-HIF- $2 \alpha$ switch evolutionarily emerges to enhance aggressive tumor growth and invasion [46]. Of note, abnormal HIF-2 $\alpha$ expression could even occur under normoxic conditions [47], which we also detected in HCC cell lines. Since non-tumor liver tissues accumulated abundant HIF$2 \alpha$ in the cytoplasm of hepatocytes, it is highly suggestive for its potential cytosolic function. In line with increased nuclear expression in tumor regions, we uncover its association with Ythdf2 gene promoter and transcriptional suppression. Intriguingly, recent work suggested that the first-line anti-HCC drug sorafenib induced HIF- $2 \alpha$ expression, whereas treating with the HIF-2 antagonist PT2385 significantly reversed sorafenib efficiency [30]. Here, we demonstrate that PT2385 rescues and requires YTHDF2 expression to exert its therapeutic effect in a HCC xenograft model. Besides transcriptional regulation, hypoxic onset allows translocation of cytosolic YTHDF2 protein to processing bodies through an unknown mechanism. In concert, PT2385 upregulates YTHDF2 expression without changing its cytosolic distribution, which optimizes YTHDF2 function in hypoxic cancer.

\section{Conclusions}

The $\mathrm{m}^{6} \mathrm{~A}$ 'reader' YTHDF2 acts as a mRNA-processing enzyme, potentially reprogramming the epitranscriptome in response to hypoxia, a frequent feature in human HCC. Our results indicate that YTHDF2 represses both tumor cells and tumor vasculature by processing IL11 and SERPINE2 mRNAs to decay. Indeed, HIF-2 $\alpha$ mediated inhibition of YTHDF2 in HCC cells provides

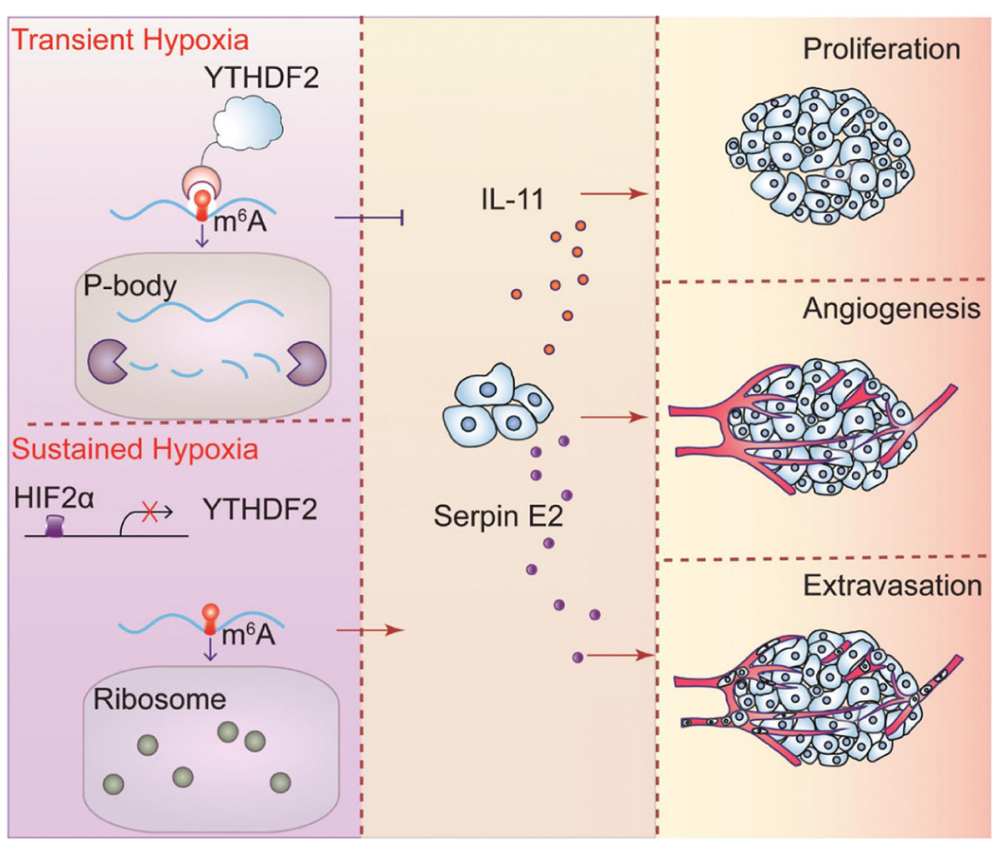

Fig. 7 Schematic model showing how hypoxia perturbs YTHDF2 to promote cancer-associated inflammation. P-body, processing body 
proof of concept that hypoxia adapts cancer epigenetics for aggressiveness, which is due, at least in part, to the stabilization of $\mathrm{m}^{6} \mathrm{~A}$-containing oncogene mRNAs. Overall, these findings highlight profound implications for understanding and targeting the $\mathrm{m}^{6} \mathrm{~A}$-methylome in human liver cancer.

\section{Materials and methods}

\section{Patient specimens}

Tumor tissues and matched adjacent non-tumor tissues were obtained from liver cancer patients who underwent liver resection at the Department of Liver Surgery, and diagnosed as HCC by the Department of Pathology, Renji Hospital, School of Medicine, Shanghai Jiaotong University. All samples were collected with informed consent, and the experiments were approved by the ethical review committee of the World Health Organization Collaborating Center for Research in Human Production (authorized by the Shanghai Municipal Government). A total number of 200 patients enrolled from 2006 to 2012 were all treatment naïve before surgery, and were routinely followed up after curative liver resection. Portions of surgically resected tissues were immediately transferred to liquid nitrogen until RNA or protein extraction. Paraffin-embedded tumors were processed into tissue microarrays for histological staining and prognostic analysis. Detailed clinicopathologic features were summarized in Additional file 2: Table S1.

\section{$\mathrm{m}^{6} \mathrm{~A}$ immunoblotting}

Poly $(\mathrm{A})^{+}$mRNA samples were denatured at $65^{\circ} \mathrm{C}$ for 5 min in 3 sample volumes of RNA incubation buffer. An equal volume of chilled $20 \times$ SSC buffer (Sigma-Aldrich) was then added before samples were spotted on the nitrocellulose membrane. After UV crosslinking for 10 min, the membrane was washed with $1 \times$ PBST buffer, blocked with $5 \%$ non-fat milk and incubated with anti$\mathrm{m}^{6} \mathrm{~A}$ antibody (1:2000, Synaptic Systems) overnight at $4{ }^{\circ} \mathrm{C}$. Then HRP-conjugated goat anti-rabbit IgG (Jackson Immuno Research Laboratories) was added to the blots for $1 \mathrm{~h}$ at room temperature and the membrane was developed with a ChemiDoc XRS system (Bio-Rad).

\section{LC-MS/MS quantification of $\mathrm{m}^{6} \mathrm{~A}$}

$100-200 \mathrm{ng}$ of mRNA was digested by nuclease P1 (2 U) in $25 \mu \mathrm{l}$ of buffer containing $25 \mathrm{mM} \mathrm{NaCl}$, and $2.5 \mathrm{mM}$ $\mathrm{ZnCl}_{2}$ at $42^{\circ} \mathrm{C}$ for $2 \mathrm{~h}$, followed by the addition of $\mathrm{NH}_{4} \mathrm{HCO}_{3}(1 \mathrm{M}, 3 \mu \mathrm{l})$ and alkaline phosphatase $(0.5 \mathrm{U})$ and incubation at $37^{\circ} \mathrm{C}$ for $2 \mathrm{~h}$. The sample was then filtered $(0.22 \mu \mathrm{m}$ pore size, $4 \mathrm{~mm}$ diameter, Millipore), and $5 \mu \mathrm{l}$ of the solution was injected into the LC-MS/MS. The nucleosides were separated by reverse- phase ultraperformance liquid chromatography on a C18 column with online mass spectrometry detection using an Agilent
6410 QQQ triple-quadrupole LC mass spectrometer in positive electrospray ionization mode. The nucleosides were quantified by using the nucleoside-to-base ion mass transitions of 282 to $150\left(\mathrm{~m}^{6} \mathrm{~A}\right)$ and 268 to 136 (A). Quantification was carried out by comparison with a standard curve obtained from pure nucleoside standards run with the same batch of samples. The $\mathrm{m}^{6} \mathrm{~A} / \mathrm{A}$ ratio was calculated based on the calibrated concentrations.

\section{$\mathrm{m}^{6} \mathrm{~A}-\mathrm{IP}$ (MeRIP)}

Total RNA was extracted from human HCC tissues or SMMC7721 cells with TRIzol (Invitrogen), then polyadenylated RNA was enriched using an Oligotex ${ }^{\circ}$ mRNA purification kit (Qiagen). In particular, additional DNase I digestion was applied to all samples to avoid DNA contamination. RNA fragmentation, $\mathrm{m}^{6} \mathrm{~A}-\mathrm{IP}$ and library preparation were proceeded according to previously published protocols [41]. Sequencing was conducted at the University of Chicago Genomics Facility on an Illumina HiSeq2500 machine in single-read mode with 50 base pairs per read. Enrichment of $\mathrm{m}^{6} \mathrm{~A}$-containing transcript segments was also analyzed through RT-qPCR. Primers targeting $\mathrm{m}^{6} \mathrm{~A}$-enriched regions of IL11 and SERPINE2 are listed: IL11_F, TCCAACAGTGAGGG TTAAGCAA; IL11_R, CCCTGAATGACAGTCCCTGC; SERPINE2_F, GGCCTCATGACAACATCGTG; SERPINE2_R, CGAGCTGCTTCTTGGTCCTG.

\section{Gene set enrichment analysis (GSEA)}

RNA-seq read counts for 14 genes that make up the hypoxia metagene signature (ALDOA, MIF, TUBB6, P4HA1, SLC2A1, PGAM1, ENO1, LDHA, CDKN3, TPI1, NDRG1, VEGFA, ACOT7 and ADM) [32] were analyzed with GSEA software v.2.0, which is available from the Broad Institute ().

\section{Animal procedures and models}

A number of $5 \times 10^{6}$ SMMC7721 or MHCC97H cells resuspended in $100 \mu \mathrm{l}$ of PBS were subcutaneously injected into the right flank of 6-week old male NCG mice (NOD-Prkd $c^{\text {scid }} I l 2 r g^{\text {null }}$ ) (Model Animal Research Center of Nanjing University). Tumor growth was monitored by caliper rule every $3-5$ days. Primary tumor volume was measured using the formula: Volume $=($ Length $\times$ Width $\left.{ }^{2}\right) / 2$. The mice were euthanized when the tumor length reached $15 \mathrm{~mm}$ or at the indicated date post tumor injection. Tumors and lungs were harvested for further histological study. For HIF-2 antagonism, PT2385 (c) was suspended in saline with $0.5 \%$ sodium carboxymethyl cellulose, $2.5 \%$ Tween 80 and $2.5 \%$ dimethyl sulfoxide. NPG mice were randomly distributed into 2 groups when the subcutaneous tumors reached an average size of $200 \mathrm{~mm}^{3}$, and administered vehicle or 
PT2385 (20 mg/kg o.p.d.) by gavage until endpoint (when the tumor size in control group reached $15 \mathrm{~mm}$ ).

Ythdf $2^{\mathrm{F} / \mathrm{F}}$ mice in C57BL/6 background were described elsewhere [48]. For liver-specific disruption, Ythdf $2^{\mathrm{F} / \mathrm{F}}$ mice were crossed with mice harboring the Cre recombinase under control of the albumin promoter to obtain the Ythdf2 $2^{\mathrm{LKO}}$ strain. Male $Y$ thdf $2^{\mathrm{F} / \mathrm{F}}$ and $Y$ thdf $2^{\mathrm{LKO}}$ littermates were injected intraperitoneally with a single dose of $25 \mathrm{mg} / \mathrm{kg}$ diethylnitrosamine (DEN) (Sigma-Aldrich) on postnatal day 14 , and 6 weeks later administrated $\mathrm{CCl}_{4}(1 \mathrm{ml} /$ $\mathrm{kg}$ ) once per week for 12 weeks. Mice were euthanized at 8.5-month old, and the livers and lungs were harvested for tumor assessment and histological experiments. The primers used for genotyping are listed: 5'Ythdf2_F, GCTTGCCTGC TACATAGTGAGA; 5'Ythdf2_R, AACTGAACTGCTTA ACCTTCTGG; 3'Ythdf2_F, GAACGGTATTGTCGGTAT TGTCA; 3'Ythdf2_R, AGACCACTCCAACACAGAACTT.

All mice were maintained under specific pathogen-free (SPF) conditions, on a $12 \mathrm{~h}$ light-dark cycle. All mouse experiments were approved by the Shanghai Administrative Committee for Laboratory Animals.

\section{YTHDF2-RIP}

The procedure was adapted from the previous protocol [49] and was described in our recent work [16]. Briefly, a total of $5 \times 10^{7}$ SMMC7721 cells expressing Flag-tagged YTHDF2 were collected and lysed in a buffer containing $150 \mathrm{mM} \mathrm{KCl}, 10 \mathrm{mM}$ HEPES (pH 7.6), $2 \mathrm{mM}$ EDTA, 0.5\% CA-630, $0.5 \mathrm{mM}$ DTT, 1:100 protease inhibitor cocktail, and $400 \mathrm{U} / \mathrm{ml}$ RNase inhibitor. Cell lysate was incubated with Flag $\mathrm{M} 2$ beads at $4{ }^{\circ} \mathrm{C}$ for $4 \mathrm{~h}$. Then the beads were washed with NT2 buffer and incubated in elution solution containing Flag peptide (Sigma-Aldrich). After washing, bound RNAs were extracted using TRIzol reagent and analyzed by $\mathrm{RT}-\mathrm{qPCR}$.

\section{RNA lifetime assay}

SMMC7721 cells were exposed to hypoxia or normoxia, and $12 \mathrm{~h}$ later actinomycin D (MCE) was added at a concentration of $5 \mathrm{mg} / \mathrm{ml}$. At the indicated time points, the cells were trypsinized and collected for RNA purification. RNA quantities were determined by RT-qPCR. The degradation rate of RNA (k) was calculated using the equation:

$$
\frac{N_{t}}{N_{0}}=e^{-k t}
$$

where $t$ is the transcription inhibition time, $k$ is the degradation rate, and $N_{t}$ and $N_{O}$ are the RNA quantities at time $\mathrm{t}$ and time 0 . The RNA lifetime $\left(t_{1 / 2}\right)$ can be calculated from the degradation rate as follows:

$$
t_{\frac{1}{2}}=\frac{\ln 2}{k}
$$

\section{Statistical analysis}

At least three biological replicates were used in each experiment unless otherwise stated. Data were analyzed with GrapPad Prism 7 and were presented as the mean \pm standard error of the mean (SEM). Comparisons between two groups were assessed using unpaired or paired (for matched comparisons) two-tailed Student's t-test, or nonparametric Mann-Whitney U-test. Multiple comparisons were assessed by one-way ANOVA. Survival rates were compared using the log-rank test. Pearson correlation coefficients $(r)$ were calculated to assess correlation and statistical significance was assessed by a two-tailed t-test of $r=0$. The statistical significance of clinicopathological differences among HCC patients were assessed by $\chi^{2}$-test.

\section{Supplementary materials and methods}

More detailed materials and methods including Cell culture, Constructs and transfections, Immunohistochemistry, Immunoblotting, Real-time quantitative PCR, Immunofluorescence, Cell proliferation, migration and mammosphere assay, Tube formation assay, Enzyme-linked immunosorbent assay, Luciferase reporter assay, Chromatin immunoprecipitation, $\mathrm{m}^{6} \mathrm{~A}$-seq data analyses are provided in Additional file 5.

\section{Supplementary information}

Supplementary information accompanies this paper at https://doi.org/10. 1186/s12943-019-1082-3.

\footnotetext{
Additional file 1: Supplementary Figures. Figure S1. Identification of a "hyper-up" pattern in m6A-epitranscriptome of human HCC. Figure S2. Expression of m6A modulators in human HCC tissues and hypoxic HCC cell lines. Figure S3. YTHDF2 deficiency enhances proliferative and proangiogenic functions of HCC cells. Figure S4. YTHDF2 inhibits tumor growth and vasculature remodeling. Figure S5. YTHDF2 deficiency upregulates IL-11 and Serpin E2 expression in HCC cells. Figure S6. YTHDF2 requires its recognitive function to degrade IL11 and SERPINE2 mRNAs. Figure S7. HIF-2a transcriptionally inhibits YTHDF2 expression in HCC cells. Figure S8. Unprocessed original scans of blots.

Additional file 2: Table S1. Clinicopathological information of $200 \mathrm{HCC}$ patients.

Additional file 3: Table S2. Upregulated genes in YTHDF2-deficient SMMC7721 cells.

Additional file 4: Table S3. Downregulated genes in YTHDF2overexpressed SMMC7721 cells.

Additional file 5: Supplementary Information.

Abbreviations

ALKBH5: a-ketoglutarate-dependent dioxygenase alkB homolog 5; CDS: Coding regions; FTO: Fat mass-and obesity-associated protein; HCC: Hepatocellular carcinoma; HIF: Hypoxia-inducible factor-2a; IL11: Interleukin 11; $\mathrm{m}^{6} \mathrm{~A}$ : $N^{6}$-methyladenosine; METTL3: Methyltransferase-like 3; PA: Plasminogen activator; RIP: RNA-immunoprecipitation; Serpin: Serinetype endopeptidase inhibitor; UTR: Untranslated regions; YTHDF2: YTH domain family 2
} 


\section{Acknowledgements}

We would like to thank all the members of the Xia, He and Smyth labs for helpful discussions and comments. We thank Prof. Ligang Wu (Shanghai Institutes for Biological Sciences, Chinese Academy of Sciences) for the suggestions on using of $3^{\prime} U T R$ reporter plasmids, and thank Dr. Luyu Yang (Department of General Surgery, Huashan Hospital, Cancer Metastasis Institute, Fudan University) for providing the HCC cell lines LM3 and MHCC97L.

\section{Authors' contributions}

J.H. conceived the project. He.Z., Zhenjun Z., J.W., Zhicong Z., B. H, J.Z. and He.Z. performed the cell and animal experiments. J.L., J.H. and Z.L. analyzed the RNA-Seq and $\mathrm{m}^{6} \mathrm{~A}$-Seq data. Zhenjun Z., Haiyan.Z., J.W. and M.F. tested the human samples and performed statistical analysis. X.H. and B.S. generated the gene knockout mice. M.J.S, Beicheng S. and Bin.S. provided important suggestions. J.H. designed the study, analyzed the data and wrote the manuscript. J.H., C.H. and Q.X. supervised the study. All authors read and approved the final manuscript.

\section{Funding}

This study was supported by National Key Research and Development Program of China (2017YFC0908102 to Q.X.), National Natural Science Foundation of China (81871970 \& 81672801 to J.H.), the Joint Program of Biomedical Engineering \& Medicine in Shanghai Jiaotong University (to J.H.), the Chen Guang Project in Shanghai Municipal Education Commission and Shanghai Education Development Foundation (15CG13 to J.H.), and The Ludwig Center at the University of Chicago Grant (to C.H.).

\section{Availability of data and materials}

The RNA-seq and MeRIP-seq data generated in this study have been deposited in the GEO database under the accession number GSE120860, GSE120659 and GSE120611. The transcriptomic profiles of SMMC7721 cells with diverse YTHDF2 expression can be found in Additional file 3: Table S2 and Additional file 4: Table S3. All the other data generated or analyzed during this study are included in the article and Additional file 5 .

\section{Ethics approval and consent to participate}

The study has been approved by the Ethics Committee of Renji Hospital. Written informed patient consent was obtained from all patients. All experimental methods abided by the Helsinki Declaration.

\section{Consent for publication}

Not applicable.

\section{Competing interests}

The authors declare no conflict of interest.

\section{Author details}

'Department of Liver Surgery, Renji Hospital, School of Medicine, Shanghai Jiaotong University, Shanghai 200127, China. ${ }^{2}$ Department of Hepatobiliary Surgery, The Affiliated Drum Tower Hospital of Nanjing University Medical School, Nanjing 210093, China. ${ }^{3}$ Department of Hepatobiliary Surgery, Sun Yat-sen University Cancer Center, Guangzhou 510060, China. ${ }^{4}$ State Key Laboratory of Oncology in South China, Sun Yat-sen University Cancer Center, Guangzhou 510060, China. ${ }^{5}$ Department of Surgery, The University of Hong Kong-Shenzhen Hospital, Shenzhen 518053, China. ${ }^{6}$ Department of Chemistry, Department of Biochemistry and Molecular Biology, Institute for Biophysical Dynamics, University of Chicago, Chicago, IL 60637, USA. ${ }^{7}$ School of Life Science and Technology, ShanghaiTech University, Shanghai 201210, China. ${ }^{8}$ Immunology of Cancer and Infection Laboratory, QIMR Berghofer Medical Research Institute, Herston, Queensland 4006, Australia. ${ }^{9}$ Key Laboratory of Reproductive Medicine, Department of Histology and Embryology, Nanjing Medical University, Nanjing 211166, China. ${ }^{10}$ Howard Hughes Medical Institute, University of Chicago, Chicago, IL 60637, USA.

Received: 28 June 2019 Accepted: 25 September 2019 Published online: 18 November 2019

\section{References}

1. Roundtree IA, Evans ME, Pan T, He C. Dynamic RNA modifications in gene expression regulation. Cell. 2017;169:1187-200.
2. Zhao BS, Roundtree IA, He C. Post-transcriptional gene regulation by mRNA modifications. Nat Rev Mol Cell Biol. 2017;18:31-42.

3. Nilsen TW. Molecular biology. Internal mRNA methylation finally finds functions. Science. 2014;343:1207-8.

4. Li Z, Weng H, Su R, Weng X, Zuo Z, Li C, Huang H, Nachtergaele S, Dong L, Hu C, et al. FTO plays an oncogenic role in acute myeloid leukemia as a N (6)-Methyladenosine RNA Demethylase. Cancer Cell. 2017;31:127-41.

5. Su R, Dong L, Li C, Nachtergaele S, Wunderlich M, Qing Y, Deng X, Wang Y, Weng $X, H u C$, et al. R-2HG exhibits anti-tumor activity by targeting FTO/m (6) a/MYC/CEBPA signaling. Cell. 2018;172:90-105 e123.

6. Zhang C, Samanta D, Lu H, Bullen JW, Zhang H, Chen I, He X, Semenza GL. Hypoxia induces the breast cancer stem cell phenotype by HIF-dependent and ALKBH5-mediated m (6) A-demethylation of NANOG mRNA. Proc Natl Acad Sci U S A. 2016;113:E2047-56.

7. Zhang S, Zhao BS, Zhou A, Lin K, Zheng S, Lu Z, Chen Y, Sulman EP, Xie K, Bogler O, et al. m (6) a Demethylase ALKBH5 maintains Tumorigenicity of Glioblastoma stem-like cells by sustaining FOXM1 expression and cell proliferation program. Cancer Cell. 2017;31:591-606 e596.

8. Chen M, Wei L, Law CT, Tsang FH, Shen J, Cheng CL, Tsang LH, Ho DW, Chiu DK, Lee JM, et al. RNA N6-methyladenosine methyltransferase-like 3 promotes liver cancer progression through YTHDF2-dependent posttranscriptional silencing of SOCS2. Hepatology. 2018;67:2254-70.

9. Barbieri I, Tzelepis K, Pandolfini L, Shi J, Millan-Zambrano G, Robson SC, Aspris D, Migliori V, Bannister AJ, Han N, et al. Promoter-bound METTL3 maintains myeloid leukaemia by m (6) A-dependent translation control. Nature. 2017;552:126-31.

10. Choe J, Lin S, Zhang W, Liu Q, Wang L, Ramirez-Moya J, Du P, Kim W, Tang S, Sliz P, et al. mRNA circularization by METTL3-elF3h enhances translation and promotes oncogenesis. Nature. 2018;561:556-60.

11. Cheng M, Sheng L, Gao Q, Xiong Q, Zhang H, Wu M, Liang Y, Zhu F, Zhang $Y$, Zhang $X$, et al. The $m(6)$ a methyltransferase METTL3 promotes bladder cancer progression via AFF4/NF-kappaB/MYC signaling network. Oncogene. 2019:38:3667-80.

12. Lin X, Chai G, Wu Y, Li J, Chen F, Liu J, Luo G, Tauler J, Du J, Lin S, et al. RNA $m$ (6) a methylation regulates the epithelial mesenchymal transition of cancer cells and translation of snail. Nat Commun. 2019;10:2065.

13. Ma JZ, Yang F, Zhou CC, Liu F, Yuan JH, Wang F, Wang TT, Xu QG, Zhou WP, Sun SH. METTL14 suppresses the metastatic potential of hepatocellular carcinoma by modulating $\mathrm{N}$ (6) -methyladenosine-dependent primary MicroRNA processing. Hepatology. 2017;65:529-43.

14. Weng H, Huang $H$, Wu H, Qin X, Zhao BS, Dong L, Shi H, Skibbe J, Shen C, Hu C, et al. METTL14 inhibits hematopoietic stem/progenitor differentiation and promotes Leukemogenesis via mRNA m (6) a modification. Cell Stem Cell. 2018;22:191-205 e199.

15. Cui $Q$, Shi H, Ye P, Li L, Qu Q, Sun G, Sun G, Lu Z, Huang Y, Yang CG, et al. $m$ (6) a RNA methylation regulates the self-renewal and tumorigenesis of Glioblastoma stem cells. Cell Rep. 2017;18:2622-34.

16. Liu J, Eckert MA, Harada BT, Liu SM, Lu Z, Yu K, Tienda SM, Chryplewicz A, Zhu AC, Yang $Y$, et al. $m$ (6) a mRNA methylation regulates AKT activity to promote the proliferation and tumorigenicity of endometrial cancer. Nat Cell Biol. 2018;20:1074-83.

17. Wang X, Lu Z, Gomez A, Hon GC, Yue Y, Han D, Fu Y, Parisien M, Dai Q, Jia $G$, et al. N6-methyladenosine-dependent regulation of messenger RNA stability. Nature. 2014;505:117-20.

18. Edupuganti RR, Geiger S, Lindeboom RGH, Shi H, Hsu PJ, Lu Z, Wang SY, Baltissen MPA, Jansen P, Rossa M, et al. N (6)-methyladenosine ( $m(6)$ a) recruits and repels proteins to regulate mRNA homeostasis. Nat Struct Mol Biol. 2017;24:870-8.

19. Zhong L, Liao D, Zhang M, Zeng C, Li X, Zhang R, Ma H, Kang T. YTHDF2 suppresses cell proliferation and growth via destabilizing the EGFR mRNA in hepatocellular carcinoma. Cancer Lett. 2019;442:252-61.

20. Paris J, Morgan M, Campos J, Spencer GJ, Shmakova A, Ivanova I, Mapperley C, Lawson H, Wotherspoon DA, Sepulveda C, et al. Targeting the RNA m (6) a reader YTHDF2 selectively compromises Cancer stem cells in acute myeloid leukemia. Cell Stem Cell. 2019.

21. Thienpont B, Steinbacher J, Zhao H, D'Anna F, Kuchnio A, Ploumakis A, Ghesquiere B, Van Dyck L, Boeckx B, Schoonjans L, et al. Tumour hypoxia causes DNA hypermethylation by reducing TET activity. Nature. 2016;537:63-8.

22. Kinnaird A, Zhao S, Wellen KE, Michelakis ED. Metabolic control of epigenetics in cancer. Nat Rev Cancer. 2016;16:694-707.

23. Intlekofer AM, Dematteo RG, Venneti S, Finley LW, Lu C, Judkins AR, Rustenburg AS, Grinaway PB, Chodera JD, Cross JR, Thompson CB. Hypoxia induces production of L-2-Hydroxyglutarate. Cell Metab. 2015;22:304-11. 
24. Fry NJ, Law BA, Ilkayeva OR, Holley CL, Mansfield KD. N (6)-methyladenosine is required for the hypoxic stabilization of specific mRNAs. RNA. 2017:23:1444-55.

25. Wilson GK, Tennant DA, McKeating JA. Hypoxia inducible factors in liver disease and hepatocellular carcinoma: current understanding and future directions. J Hepatol. 2014;61:1397-406.

26. Chaisaingmongkol J, Budhu A, Dang H, Rabibhadana S, Pupacdi B, Kwon SM, Forgues M, Pomyen Y, Bhudhisawasdi V, Lertprasertsuke N, et al. Common molecular subtypes among Asian hepatocellular carcinoma and Cholangiocarcinoma. Cancer Cell. 2017;32:57-70 e53.

27. Yuan JH, Yang F, Wang F, Ma JZ, Guo YJ, Tao QF, Liu F, Pan W, Wang $\Pi$, Zhou CC, et al. A long noncoding RNA activated by TGF-beta promotes the invasion-metastasis cascade in hepatocellular carcinoma. Cancer Cell. 2014;25:666-81.

28. Wagenblast E, Soto M, Gutierrez-Angel S, Hartl CA, Gable AL, Maceli AR, Erard N, Williams AM, Kim SY, Dickopf S, et al. A model of breast cancer heterogeneity reveals vascular mimicry as a driver of metastasis. Nature. 2015;520:358-62

29. Chen W, Hill H, Christie A, Kim MS, Holloman E, Pavia-Jimenez A, Homayoun F, Ma Y, Patel N, Yell P, et al. Targeting renal cell carcinoma with a HIF-2 antagonist. Nature. 2016;539:112-7.

30. $\mathrm{Xu} J$, Zheng $L$, Chen J, Sun $Y$, Lin H, Jin RA, Tang M, Liang $X$, Cai $X$. Increasing AR by HIF-2alpha inhibitor (PT-2385) overcomes the side-effects of sorafenib by suppressing hepatocellular carcinoma invasion via alteration of pSTAT3, pAKT and pERK signals. Cell Death Dis. 2017:8:e3095.

31. Courtney KD, Infante JR, Lam ET, Figlin RA, Rini BI, Brugarolas J, Zojwalla NJ, Lowe AM, Wang K, Wallace EM, et al. Phase I dose-escalation trial of PT2385, a first-in-class hypoxia-inducible factor-2alpha antagonist in patients with previously treated advanced clear cell renal cell carcinoma. J Clin Oncol. 2018;36:867-74.

32. Buffa FM, Harris AL, West CM, Miller CJ. Large meta-analysis of multiple cancers reveals a common, compact and highly prognostic hypoxia metagene. Br J Cancer. 2010;102:428-35.

33. Qiu GZ, Jin MZ, Dai JX, Sun W, Feng JH, Jin WL. Reprogramming of the tumor in the hypoxic niche: the emerging concept and associated therapeutic strategies. Trends Pharmacol Sci. 2017;38:669-86.

34. Rankin EB, Giaccia AJ. Hypoxic control of metastasis. Science. 2016;352:175-80.

35. Goel S, Wong AH, Jain RK. Vascular normalization as a therapeutic strategy for malignant and nonmalignant disease. Cold Spring Harb Perspect Med. 2012;2:a006486

36. Bollrath J, Phesse TJ, von Burstin VA, Putoczki T, Bennecke M, Bateman T, Nebelsiek T, Lundgren-May T, Canli O, Schwitalla S, et al. gp130-mediated Stat3 activation in enterocytes regulates cell survival and cell-cycle progression during colitis-associated tumorigenesis. Cancer Cell. 2009;15:91-102.

37. Bajou K, Peng H, Laug WE, Maillard C, Noel A, Foidart JM, Martial JA, DeClerck YA. Plasminogen activator inhibitor-1 protects endothelial cells from FasL-mediated apoptosis. Cancer Cell. 2008;14:324-34.

38. Valiente M, Obenauf AC, Jin X, Chen Q, Zhang XH, Lee DJ, Chaft JE, Kris MG, Huse JT, Brogi E, Massague J. Serpins promote cancer cell survival and vascular co-option in brain metastasis. Cell. 2014;156:1002-16.

39. Buchholz M, Biebl A, Neesse A, Wagner M, Iwamura T, Leder G, Adler G, Gress TM. SERPINE2 (protease nexin I) promotes extracellular matrix production and local invasion of pancreatic tumors in vivo. Cancer Res. 2003;63:4945-51.

40. Zhu T, Roundtree IA, Wang P, Wang X, Wang L, Sun C, Tian Y, Li J, He C, Xu $Y$. Crystal structure of the YTH domain of YTHDF2 reveals mechanism for recognition of N6-methyladenosine. Cell Res. 2014;24:1493-6.

41. Dominissini D, Moshitch-Moshkovitz S, Schwartz S, Salmon-Divon M, Ungar L, Osenberg S, Cesarkas K, Jacob-Hirsch J, Amariglio N, Kupiec M, et al. Topology of the human and mouse m6A RNA methylomes revealed by m6A-seq. Nature. 2012;485:201-6.

42. Huang $H$, Weng $H$, Sun W, Qin X, Shi H, Wu H, Zhao BS, Mesquita A, Liu C, Yuan CL, et al. Recognition of RNA N (6)-methyladenosine by IGF2BP proteins enhances mRNA stability and translation. Nat Cell Biol. 2018;20:285-95.

43. Llovet JM, Montal R, Sia D, Finn RS. Molecular therapies and precision medicine for hepatocellular carcinoma. Nat Rev Clin Oncol. 2018.

44. De Palma M, Biziato D, Petrova TV. Microenvironmental regulation of tumour angiogenesis. Nat Rev Cancer. 2017;17:457-74.

45. Koh MY, Powis G. Passing the baton: the HIF switch. Trends Biochem Sci. 2012;37:364-72
46. Koh MY, Lemos R Jr, Liu X, Powis G. The hypoxia-associated factor switches cells from HIF-1alpha- to HIF-2alpha-dependent signaling promoting stem cell characteristics, aggressive tumor growth and invasion. Cancer Res. 2011;71:4015-27.

47. Mutvei AP, Landor SK, Fox R, Braune EB, Tsoi YL, Phoon YP, Sahlgren C, Hartman J, Bergh J, Jin S, Lendahl U. Notch signaling promotes a HIF2alphadriven hypoxic response in multiple tumor cell types. Oncogene. 2018.

48. Li Z, Qian P, Shao W, Shi H, He XC, Gogol M, Yu Z, Wang Y, Qi M, Zhu Y, et al. Suppression of $m(6)$ a reader $Y$ thdf2 promotes hematopoietic stem cell expansion. Cell Res. 2018;28:904-17.

49. Peritz T, Zeng F, Kannanayakal TJ, Kilk K, Eiriksdottir E, Langel U, Eberwine J. Immunoprecipitation of mRNA-protein complexes. Nat Protoc. 2006:1:577-80.

\section{Publisher's Note}

Springer Nature remains neutral with regard to jurisdictional claims in published maps and institutional affiliations.
Ready to submit your research? Choose BMC and benefit from:

- fast, convenient online submission

- thorough peer review by experienced researchers in your field

- rapid publication on acceptance

- support for research data, including large and complex data types

- gold Open Access which fosters wider collaboration and increased citations

- maximum visibility for your research: over $100 \mathrm{M}$ website views per year

At BMC, research is always in progress.

Learn more biomedcentral.com/submissions 